\title{
Inhibition of p600 Expression Suppresses Both Invasiveness and Anoikis Resistance of Gastric Cancer
}

\author{
Hiroshi Sakai, MD ${ }^{1}$, Kenoki Ohuchida, MD, $\mathrm{PhD}^{1,2}$, Kazuhiro Mizumoto, MD, $\mathrm{PhD}^{1,3}$, Lin Cui, $\mathrm{MD}^{1}$, \\ Kohei Nakata, MD, PhD ${ }^{1}$, Hiroki Toma, MD, PhD $^{1}$, Eishi Nagai, MD, PhD ${ }^{1}$, and Masao Tanaka, MD, PhD, FACS ${ }^{1}$ \\ ${ }^{1}$ Departments of Surgery and Oncology, Graduate School of Medical Sciences, Kyushu University, Fukuoka, Japan; \\ ${ }^{2}$ Department of Advanced Medical Initiatives, Graduate School of Medical Sciences, Kyushu University, Fukuoka, Japan; \\ ${ }^{3}$ Kyushu University Hospital Cancer Center, Fukuoka, Japan
}

\begin{abstract}
Background. Advanced gastric cancers often metastasize to distant organs and the peritoneum, leading to a poor prognosis. Both invasiveness and resistance to anchorageindependent cell death (anoikis) are important factors in the process of metastasis. p600 (600-kDa protein), recently identified from a cervical cancer cell line, plays a role in both anoikis resistance and cell migration. In this study, we examined whether p600 is involved in the progression of gastric cancer.
\end{abstract}

Methods. We used both normal gastric mucosal cells and cancer cells laser-microdissected from 42 gastric cancers and their normal counterparts, and compared their p600 mRNA expression levels with quantitative reverse transcriptase-polymerase chain reaction. We inhibited p600 expression in two gastric cancer cell lines with siRNA and examined its effect on the invasiveness and anoikis resistance both in vitro and in vivo.

Results. Expression of p600 mRNA was significantly higher in gastric cancer cells than in normal mucosal cells $(P=0.027)$. The invasion assay revealed that invasiveness was significantly reduced by inhibition of p600 $(P<0.01)$. In vitro experiments revealed that cell viability and colonyformation capacity under anchorage-independent conditions were significantly reduced by inhibition of p600 $(P<0.05)$. In vivo experiments also showed that the

(C) The Author(s) 2011. This article is published with open access at Springerlink.com

First Received: 21 April 2010;

Published Online: 24 February 2011

K. Ohuchida, MD, PhD

e-mail: kenoki@surg1.med.kyushu-u.ac.jp establishment of intraperitoneal disseminated tumors was significantly suppressed by transient inhibition of p600 $(P<0.001)$.

Conclusions. Our results strongly suggest that p600 is involved in gastric cancer progression, and has a potential to be a new molecular target for gastric cancer therapy.

Gastric cancer is the fourth most common malignancy, and the second most common cause of cancer mortality worldwide. ${ }^{1}$ Although the proportion of early gastric cancers is increasing around the world, many patients still present with advanced-stage disease. ${ }^{2}$ The median survival of patients with metastatic or unresectable advanced gastric cancer ranges from 6 to 9 months. ${ }^{3,4}$ The control of advanced gastric cancer remains difficult, despite advances in chemotherapy and radiotherapy. ${ }^{5,6}$ Therefore, new therapies are needed to improve the 5-year survival rate of patients with advanced gastric cancer.

Metastasis is a complex process. ${ }^{7}$ Gastric cancer cells need to migrate to and invade through the basement membrane, and enter the abdominal cavity or the circulatory/lymphatic systems. They need to survive without contacting the extracellular matrix (ECM), invade either the peritoneum or circulatory/lymphatic systems, and then proliferate in a foreign environment. ${ }^{8}$ Normal epithelial cells undergo apoptosis after losing contact with neighboring cells or the ECM. This process is termed anoikis. ${ }^{9}$ Gastric cancer cells have gained mechanisms to resist anoikis, and thereby survive and travel through the abdominal cavity or the lymphatic/circulatory systems, without the need for ECM contact. ${ }^{8}$ Therefore, invasiveness and resistance to anoikis are critical for the systemic spread of cancer cells. Despite the importance of 
invasiveness and anoikis resistance in the progression of cancer, the molecular mechanisms that underlie this process have yet to be fully identified.

Recently, p600 (600-kDa protein) was isolated from cervical carcinoma cells as a retinoblastoma binding protein. ${ }^{10,11}$ p600 is localized in both the nuclear and the cytoplasmic compartments. ${ }^{11}$ In the nucleus, p600 localizes to the nuclear matrix components and interacts with retinoblastoma binding protein. ${ }^{11}$ In the cytoplasm, p600 accumulates at the leading edge of the cell membrane, and plays an essential role in membrane morphogenesis, which is crucial for cell migration. ${ }^{11}$ p600 has also been shown to play a critical role in anchorage-independent growth by binding to viral oncoproteins such as human papillomavirus 16 E7 (HPV-16 E7) and bovine papillomavirus 1 E7 (BPV-1 E7). ${ }^{10,12,13}$ Inhibition of p600 expression suppresses anchorage-independent growth of HPV-16-positive Caski cervical cancer cells, HPV-16 E7-expressing NIH 3T3 fibroblasts, and BPV-1 E6- and E7-expressing C127 murine cells. $^{10,12,13}$ Furthermore, anchorage-independent cell growth was suppressed by inhibition of p600 expression in U2OS human osteosarcoma cells and NIH 3T3 fibroblasts, which do not contain viral oncogene sequences, without affecting their growth in monolayer culture. ${ }^{10}$ Several reports also showed that p600 was upregulated after transformation of mouse embryonic fibroblasts, and upregulated in a metastatic tumor cell line in a xenogeneic breast cancer model. ${ }^{14,15}$ Therefore, there is a possibility that p600 correlates with metastatic potential in other solid tumors.

In this study, we examined whether p600 expression was higher in gastric cancer cells than in normal gastric mucosal cells, and investigated the effect of p600 inhibition on the invasiveness and anchorage-independent growth of gastric cancer cells in vitro. Finally, we examined whether inhibition of p600 prevented the establishment of disseminated gastric tumors in vivo. Our results suggest that p600 is involved in gastric cancer progression and has the potential to be a new molecular target for gastric cancer therapy.

\section{MATERIALS AND METHODS}

\section{Clinical Samples}

Forty-two patients with gastric cancer underwent surgical resection at our institute. Experienced pathologists performed histological examination of all samples, including tissues from organs adjacent to the gastric specimens. The study was approved by the ethics committee of Kyushu University and conducted according to the Ethical Guidelines for Human Genome/Gene Research enacted by the Japanese government.

\section{Laser Microdissection}

Frozen tissue samples were cut into $8-\mu \mathrm{m}$ sections, and one section was stained with hematoxylin and eosin for histologic examination. Cancer lesions and normal gastric mucosal lesions were selectively isolated with a laser microdissection and pressure catapulting system (PALM Microlaser Technologies, Bernried, Germany) in accordance with the manufacturer's protocols. After microdissection, total RNA was extracted from the selected cells and subjected to quantitative real-time reverse transcriptase-polymerase chain reaction (qRT-PCR) to measure p600 mRNA levels as previously described. ${ }^{16,17}$

\section{Quantitative Real-Time Reverse Transcriptase- Polymerase Chain Reaction}

We designed specific primers as follows: $p 600,5^{\prime}$-CAC ACATCCTCCACCAAGTG-3' (forward) and 5'-GGGCC CGAAGTAGTGTGATA-3' (reverse); 18S rRNA, 5'-GT AACCCGTTGAACCCCATT-3' (forward) and $5^{\prime}$-CCATC CAATCGGTAGTAGCG-3' (reverse). One-step qRT-PCR was performed with a QuantiTect SYBR Green RT-PCR kit (Qiagen, Tokyo, Japan) with a Chrom4 Real-Time PCR Detection System (Bio-Rad Laboratories, Hercules, CA) as previously described. ${ }^{18}$ Each sample was run in triplicate, and the expression of $p 600$ mRNA was presented as the ratio between the expression of $p 600$ mRNA and that of $18 \mathrm{~S}$ rRNA.

\section{Cells Lines}

Human gastric cancer cell lines (MKN45, MKN74, KATO III) were purchased from the Japanese Collection of Research Bioresources (Osaka, Japan). AGS, MKN7, and NCI-N87 were the gift of the Department of Cancer Therapy and Research Graduate School of Medical Sciences, Kyushu University. Cells were cultured in RPMI medium supplemented with streptomycin $(100 \mu \mathrm{g} / \mathrm{mL})$, penicillin $(100 \mathrm{U} / \mathrm{mL})$, and $10 \%$ fetal bovine serum (FBS) at $37^{\circ} \mathrm{C}$ in a humidified $90 \%$ air and $10 \% \mathrm{CO}_{2}$ atmosphere.

\section{Transfections}

Cells were transfected with p600-targeting small interfering RNA (siRNA) (sense, ggaaagaacaucauuguuaTT; antisense, uaacaaugauguucuuuccTT) (B-Bridge, Mountain View, CA), or negative control siRNA (B-Bridge), which was confirmed not to target a specific array by electroporation with a Nucleofector System (Amaxa Biosystems, Koln, Germany) according to the manufacturer's instructions. 


\section{Western Blot Analysis}

Cells or tissues were lysed in Pro-Prep (iNtRON Biotechnology, Seongnam, Korea). Cell or tissue lysate proteins $(20-50 \mu \mathrm{g})$ were fractionated on $4-15 \%$ sodium dodecyl sulfate-polyacrylamide gel electrophoresis gels (Bio-Rad Laboratories) and transferred to a polyvinylidene diflouride membrane (Millipore, Billerica, MA). The membrane was incubated overnight at $4^{\circ} \mathrm{C}$ with anti-p600 (ab86738; Abcam, Cambridge, UK; 1:2500), anti-matrix metalloproteinase (MMP)-2 (sc-10736; Santa Cruz Biotechnology, Santa Cruz, CA; 1:200), anti-MMP-7 (sc80205; 1:200), anti-MMP-9 (EP1254; Millipore; 1:1000), anti-urokinase-type plasminogen activator (uPA) (sc-6830; 1:200), anti-E-cadherin (\#4065; Cell Signaling Technology, Boston, MA; 1:1000) or anti- $\beta$-actin (sc-1616; 1:2000) antibodies and then probed with secondary antibodies conjugated to horseradish peroxidase (Santa Cruz Biotechnology). Immunoblots were detected by enhanced chemiluminescence with ChemiDoc XRS (Bio-Rad Laboratories).

\section{In Vitro Invasion Assay}

Invasiveness of cancer cells was evaluated by counting the number of cells invading through a Matrigel-coated transwell as previously described. ${ }^{19}$ Briefly, transwell inserts with $8-\mu \mathrm{m}$ pores were coated with Matrigel $(20 \mu \mathrm{g} /$ well; BD, Bedford, MA), and $1 \times 10^{5}$ cells were seeded into the Matrigel-coated transwell insert. After $48 \mathrm{~h}$ of incubation, cells that had invaded to the lower surface of the Matrigel-coated membrane were counted under a light microscope. Each experiment was carried out in triplicate wells, and independent experiments were repeated at least three times.

\section{Cell Proliferation Assay}

Cells $\left(1 \times 10^{4} /\right.$ well $)$ were seeded in 24 -well tissue culture plates (Becton Dickinson Labware, Bedford, MA) and incubated. The number of cells was investigated at the indicated time points by measuring the fluorescence intensity of propidium iodide as previously described. ${ }^{20}$ All experiments were performed in triplicate wells and were repeated at least three times.

\section{Apoptosis Assay and Anoikis Assay}

Cells $\left(1 \times 10^{4} /\right.$ well $)$ were suspended in 24 -well tissue culture plates (Becton Dickinson Labware) for the apoptosis assay. For the anoikis assay, cells were suspended $\left(2 \times 10^{4} /\right.$ well $)$ in 24 -well ultra-low-attachment plates, the surfaces of which consist of a covalently bound hydrogel layer that is hydrophilic and electrically neutral (Corning, Corning, NY). After incubation for 3 days, cells $\left(1 \times 10^{6}\right)$ were pooled and stained with Annexin V-FITC (MACS, Miltenyi Biotec) followed by propidium iodide. Labeled cells were analyzed with an Epics Altra flow cytometer (Beckman Coulter).

\section{Colony-Formation Assay Under Anchorage-Dependent Conditions}

Cells $\left(1 \times 10^{3} /\right.$ dish $)$ were suspended and incubated. After 14 days, the number of colonies was counted with a ChemiDoc XRS System (Bio-Rad Laboratories). All experiments were performed in triplicate wells and were repeated at least three times.

\section{Floating Culture and Evaluation of Cell Viability}

Cells $\left(2 \times 10^{4} /\right.$ well $)$ were seeded in 24 -well ultra-lowattachment plates (Corning) and incubated. The number of cells was investigated at the indicated time points by measuring the fluorescence intensity of propidium iodide as previously described. ${ }^{20}$ All experiments were performed in triplicate wells and were repeated at least three times.

\section{Soft Agar Colony-Formation Assay}

Cells $\left(1 \times 10^{3} /\right.$ well in a 6 -well plate $)$ were suspended in RPMI 1640 ( $1 \mathrm{~mL}$, supplemented with $20 \%$ FBS and $0.33 \%$ agar) and plated over a layer of solidified RPMI 1640 medium $(1.5 \mathrm{~mL}$, supplemented with $20 \%$ FBS and $0.66 \%$ agar). After incubation for 28 days, the number of colonies was counted with a ChemiDoc XRS System (Bio-Rad Laboratories). All experiments were performed in triplicate wells and were repeated at least three times.

\section{In Vivo Studies}

MKN45 cells $\left(5 \times 10^{5}\right)$ transfected with p600-targeting siRNA or with control siRNA were injected into the abdominal cavity of the 25-week-old female SCID mice (C.B-17/IcrCrj-scid; Kyudo, Tosu, Japan) along with $100 \mu \mathrm{L}$ of RPMI containing 10\% FBS. After 4 weeks, mice were humanely killed, and any disseminated nodules of $>1 \mathrm{~mm}$ in diameter were resected and counted.

\section{Statistical Analysis}

Statistical analysis was performed by JMP7 for Windows software. All data are expressed as mean \pm standard deviation. Differences between groups were 
FIG. 1 Gastric cancer cells express high levels of p600 mRNA. a Expression levels of p600 mRNA and protein in gastric cancer tissues and normal gastric tissues. (i) Reverse transcriptase-polymerase chain reaction (RT-PCR). (ii) Western blot test. $\beta$-Actin was used as the internal control for immunoblotting. The expression level of p600 protein was consistent with the expression level of $p 600$ mRNA, and there was a tendency for gastric cancer tissues to have higher expression of p600 than normal gastric tissues. b Expression levels of p600 mRNA in laser-microdissected gastric cancer cells and normal gastric mucosal cells was determined by quantitative RT-PCR $(n=42)$. Gastric cancer cells showed significantly higher expression levels of $p 600$ mRNA than normal gastric mucosal cells $(P=0.027)$. c Expression levels of $p 600$ mRNA and protein in gastric cancer cell lines and normal gastric mucosal cells obtained from surgically resected normal gastric mucosa. (i) RT-PCR. (ii) Western blot test. $\beta$-Actin was used as the internal control for immunoblotting. Gastric cancer cell lines showed higher levels of p600 expression than normal gastric mucosal cells

estimated by Student's $t$-test and/or Spearman's rank correlation, and repeated measures were assessed by analysis of variance. Differences were considered significant at $P<0.05$.

\section{RESULTS}

\section{Gastric Cancer Cells Express High Levels of p600 $m R N A$}

We examined the expression level of p600 mRNA and protein using bulk tissues from five patients with gastric cancer. The expression level of $\mathrm{p} 600$ protein was consistent with the expression level of $p 600$ mRNA, and there was a tendency for gastric cancer tissues to have higher expression of p600 mRNA and protein than normal gastric tissues (Fig. 1a). To examine whether $p 600$ mRNA expression was higher in gastric cancer cells than in normal gastric mucosal cells, we measured p600 mRNA expression in both lasermicrodissected gastric cancer cells and normal gastric mucosal cells. $p 600$ mRNA was significantly upregulated in gastric cancer cells compared with normal gastric mucosal cells (Fig. $1 \mathrm{~b} ; P=0.027$ ). We also investigated the expression of p600 in a panel of gastric cancer cell lines. For comparison, we used normal gastric mucosal cells obtained from surgically resected normal gastric mucosa. We found that all gastric cancer cell lines showed higher levels of p600 expression than normal mucosal cells (Fig. 1c).

\section{Effect of p600 Inhibition on the Invasiveness of Gastric Cancer Cells}

p600 knockdown affects cell membrane ruffle formation, which is linked to cell mobility. ${ }^{11}$ We examined the effect of p600 inhibition on the invasiveness of gastric cancer cells. To inhibit the expression of p600, we used p600-targeting siRNA, and we found that p600 expression
(a) Expression of $p 600$
(i) $\frac{7}{5}$ mRNA (/18s rRNA)

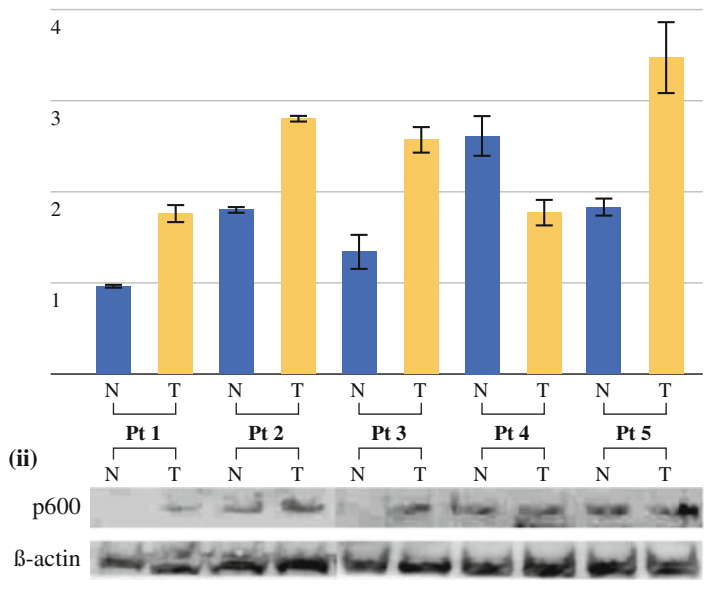

(b) Expression of $p 600$

\begin{tabular}{l} 
(b) $\begin{array}{l}\text { Expression of } p 600 \\
\text { mRNA (/18s rRNA) }\end{array}$ \\
\hline $6 \quad p=0.027$
\end{tabular}

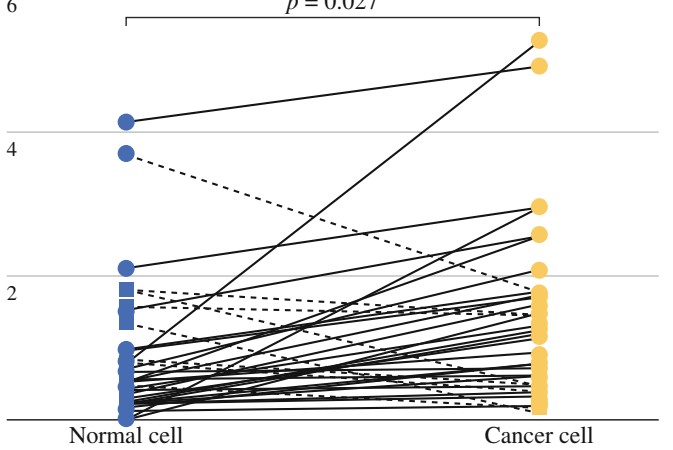

(c) Expression of $p 600$ (i) $\frac{}{5}$ mRNA (/18s rRNA)

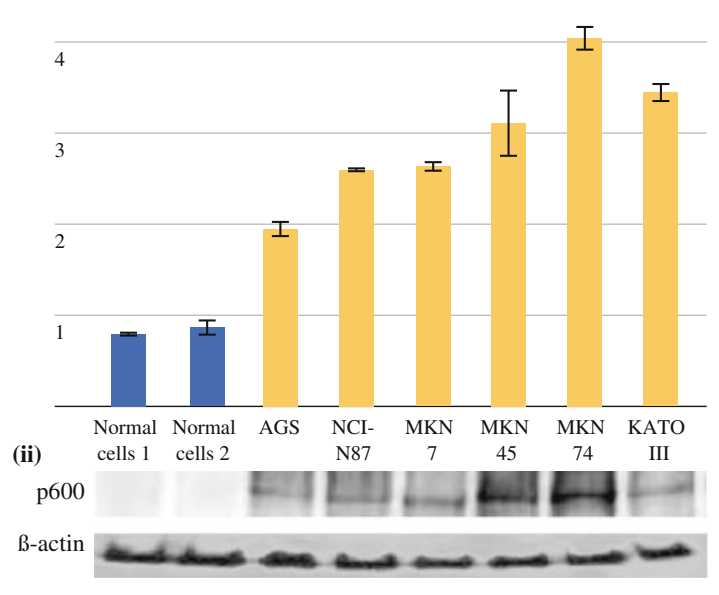

was significantly inhibited for 5 days after transfection with p600-targeting siRNA (Fig. 2a, b). The number of invading MKN45 and AGS cells was significantly decreased by p600 knockdown (Fig. 2c, d; MKN45, $P=0.004$; AGS, $P<0.001)$. 
(a) Expression of $p 600$

mRNA (/Control)

MKN45

(i) $\frac{\mathrm{m}}{1.2}$
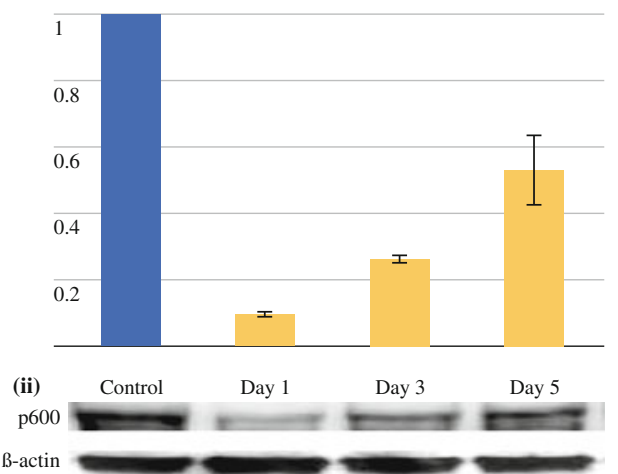

(c)

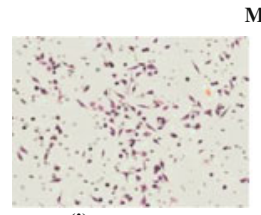

MKN45

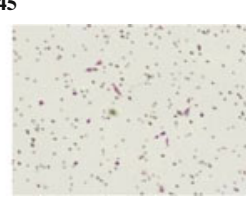

(i) Contro

(ii) $\mathrm{p} 600$ siRNA

(iii) Invaded

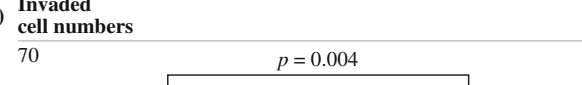

70

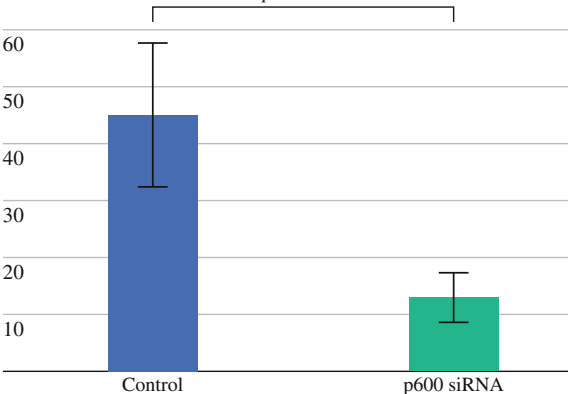

(e)

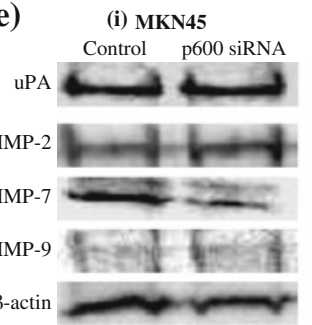

(ii) AGS

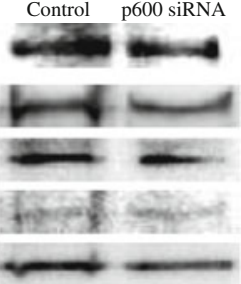

FIG. 2 Inhibition of p600 reduces the invasiveness of gastric cancer cells. a Effect of p600-targeting siRNA in the MKN45 gastric cancer cell line. (i) Reverse transcriptase-polymerase chain reaction (RTPCR). (ii) Western blot test. b Effect of p600-targeting siRNA in the AGS gastric cancer cell line. (i) RT-PCR. (ii) Western blot test. $\beta$-Actin was used as the internal control for immunoblotting. p600 expression in both gastric cancer cell lines was inhibited by p600-targeting siRNA for at least 5 days. c (i) Microscopic appearance of the invading control siRNA-transfected MKN45 cells, and (ii) p600-targeting siRNAtransfected MKN45 cells. (iii) Number of invaded cells. d (i) Microscopic appearance of invading control siRNA-transfected AGS cells, and (ii) p600-targeting siRNA-transfected AGS cells. (iii) Number of (b) Expression of $p 600$
AGS

(i) $\frac{\mathbf{m}}{1.2}$

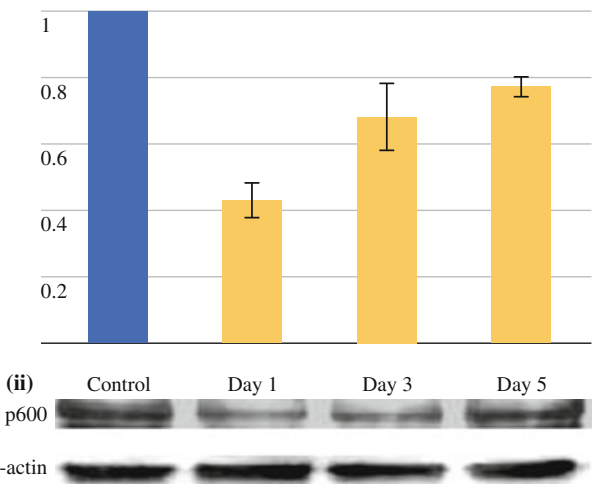

(d)

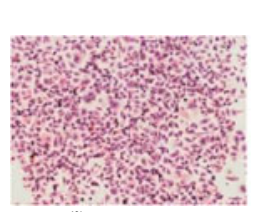

(i) Control
AGS

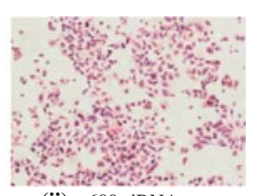

(ii) $\mathrm{p} 600$ siRNA (iii) Invaded

Invaded
cell numbers
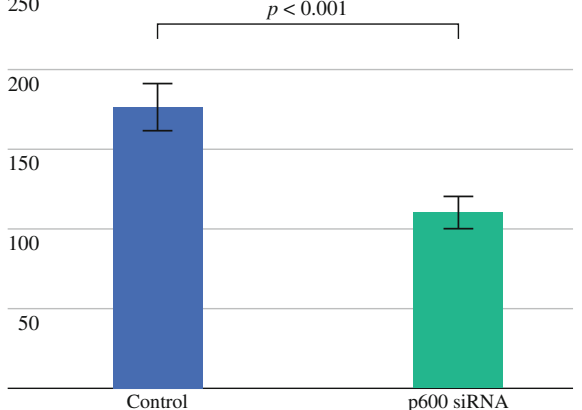

(f)

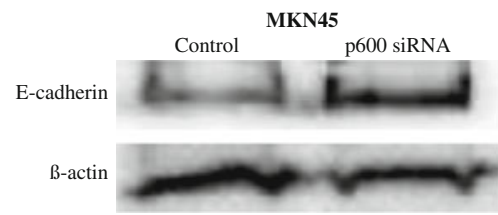

invaded cells. Data are expressed as mean \pm SD. Inhibition of p600 reduced the invasiveness of both gastric cancer cell lines (MKN45, $P=0.004$; AGS, $P<0.001)$. e Western blot analysis of urokinasetype plasminogen activator (uPA), matrix metalloproteinase (MMP)-2, MMP-7, and MMP-9 expression levels. (i) MKN45. (ii) AGS. $\beta$-Actin was used as the internal control for immunoblotting. MMP-7 expression was decreased by inhibition of p600 in both gastric cancer cell lines. f Western blot analysis of E-cadherin expression in MKN45 gastric cancer cells. $\beta$-Actin was used as the internal control for immunoblotting. Expression of E-cadherin was higher in p600-targeting siRNAtransfected MKN45 cells than in control siRNA-transfected MKN45 cells 
FIG. 3 Inhibition of p600 does not affect cell proliferation, apoptosis, and colony-formation capacity under anchorage-dependent conditions. a Proliferation assay under anchorage-dependent conditions. There was no significant difference between p600-targeting siRNA-transfected MKN45 cells and control siRNA-transfected MKN45 cells in terms of cell proliferation under anchorage-dependent conditions. b Proportion of apoptotic cells in (i) control siRNAtransfected MKN45 cells and (ii) p600-targeting siRNA-transfected MKN45 cells as analyzed by flow cytometry. There was no significant difference between p600-targeting siRNA-transfected MKN45 cells and control siRNA-transfected MKN45 cells in terms of apoptotic cells proportion. $\mathbf{c}$ Macroscopic appearance of colonies formed under anchorage-dependent conditions; (i) control siRNA-transfected MKN45 cells and (ii) p600-targeting siRNA-transfected MKN45 cells. (iii) Data graph. Data are expressed as mean \pm SD. There was no significant difference between $p 600$-targeting siRNA-transfected MKN45 cells and control siRNA-transfected MKN45 cells in terms of colony-formation capacity under anchorage-dependent conditions

Some reports show that MMP-2, MMP-7, MMP-9, and uPA affect gastric cancer invasion. ${ }^{21-24}$ Therefore, we examined the expression of these three MMPs and uPA. Although we observed no difference in the expression levels of MMP-2, MMP-9, and uPA between p600-targeting siRNA-transfected cells and control siRNA-transfected cells, the expression level of MMP-7 was decreased by inhibition of p600 in both gastric cancer cell lines (MKN45 and AGS; Fig. 2e). A previous report shows that the expression of E-cadherin is decreased by MMP-7. ${ }^{25}$ Therefore, we also examined the expression of E-cadherin. The results showed that E-cadherin was overexpressed in MKN45 cells by inhibition of p600 (Fig. 2f).

\section{Effect of p600 Inhibition on Anchorage-Dependent Cell} Proliferation, Apoptosis, and Colony-Formation Capacity of Gastric Cancer Cells

p600 did not affect the growth of U2OS human osteosarcoma cells in monolayer culture. ${ }^{10}$ Therefore, we examined the effect of p600 inhibition on anchoragedependent cell proliferation, apoptosis rate, and colonyformation capacity of gastric cancer cells in monolayer culture. Inhibition of p600 did not affect cell proliferation, apoptosis rate, or colony-formation capacity of MKN45 gastric cancer cells (Fig. 3a-c). Similar results were obtained with AGS gastric cancer cells (data not shown).

\section{Inhibition of p600 Sensitizes Gastric Cancer Cells to Anoikis and Decreases Cell Viability and Colony- Formation Capacity Under Anchorage-Independent Conditions}

We examined the effect of p600 inhibition on anchorage-independent cell viability and sensitivity to anoikis in
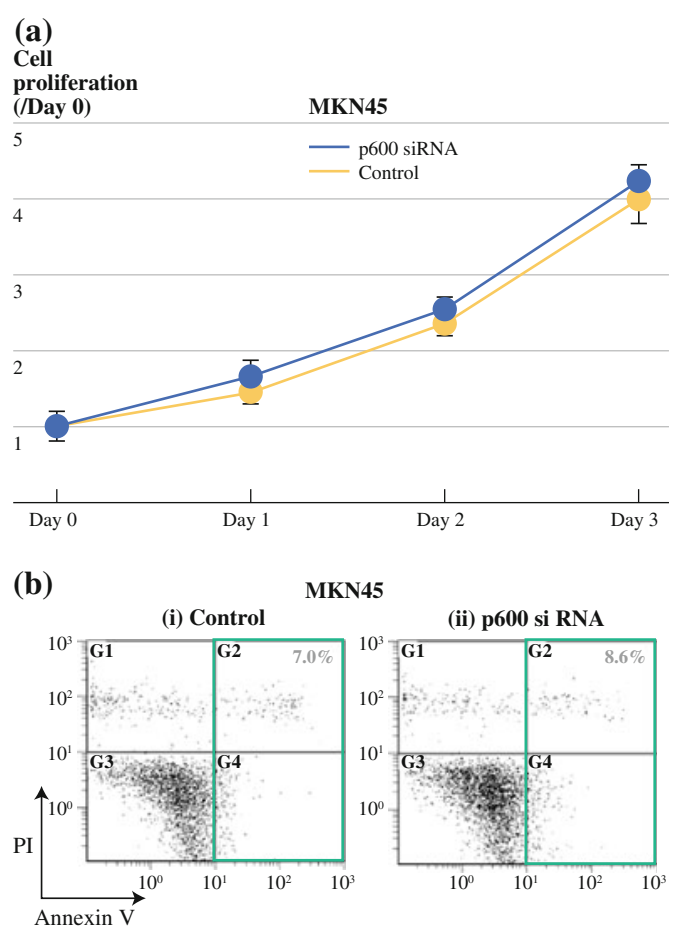

(c)

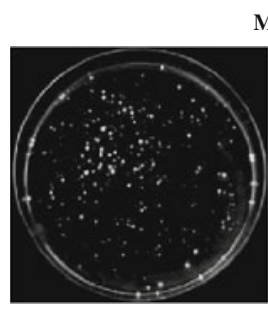

(i) Control
MKN45

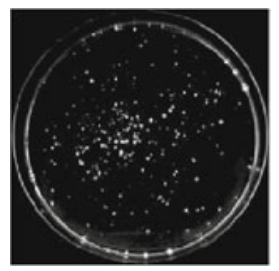

(ii) p600 si RNA (iii) Colony numbers

400

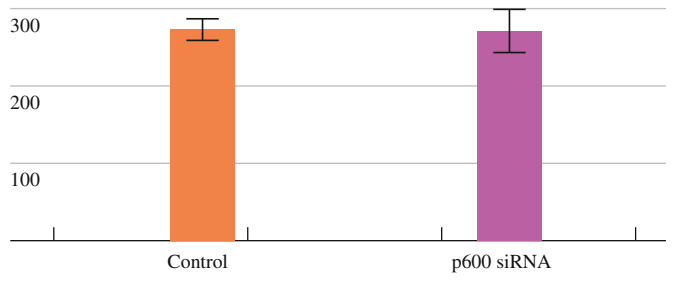

gastric cancer cells. Inhibition of p600 significantly reduced cell viability (Fig. 4a; MKN45, $P=0.033$; AGS, $P<0.001)$ and sensitized both MKN45 and AGS cells to anoikis (Fig. 4b, c). Furthermore, we investigated the effects of p600 inhibition on the anchorage-independent colony-formation capacity of MKN45 and AGS cells, and we found that the colony-formation capacity of cells transfected with p600-targeting siRNA was significantly reduced compared with that of cells transfected with control siRNA under anchorage-independent conditions (Fig. 4d, e; MKN45, $P<0.001$; AGS, $P=0.003$ ). 

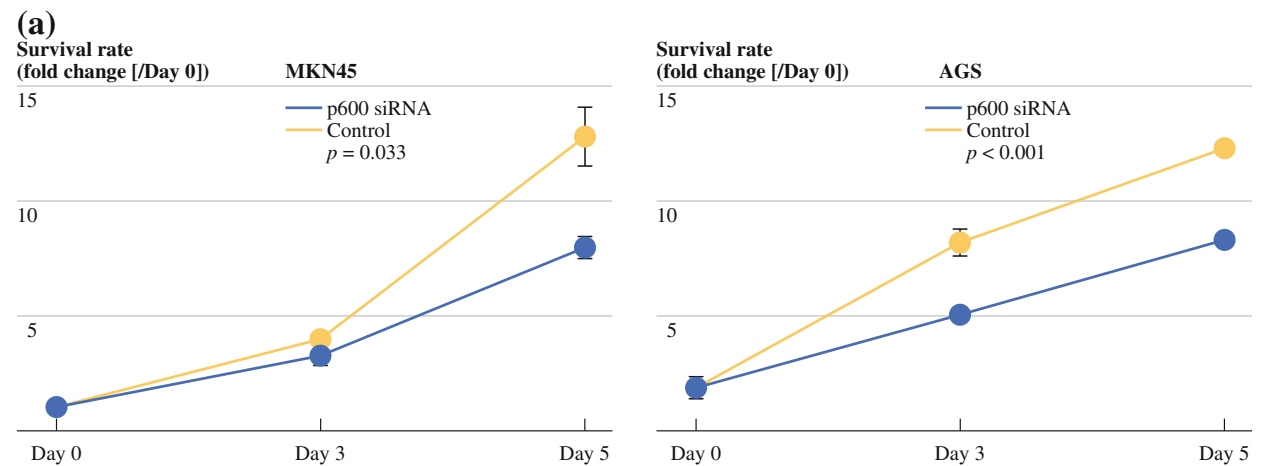

(b)

MKN45

(c)
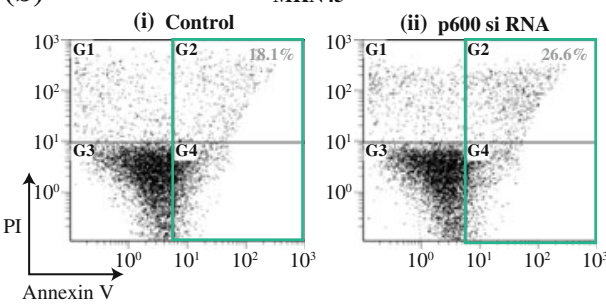

AGS
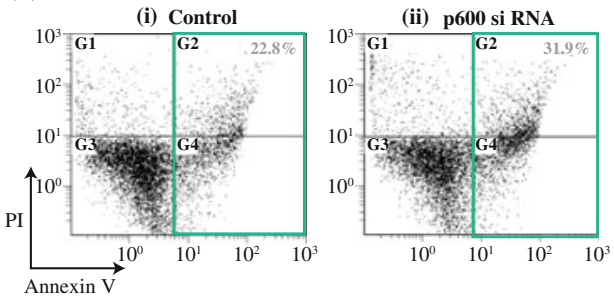

(d)

$\begin{array}{lll}\text { (i) Control MKN45 (ii) p600 si RNA } & \end{array}$
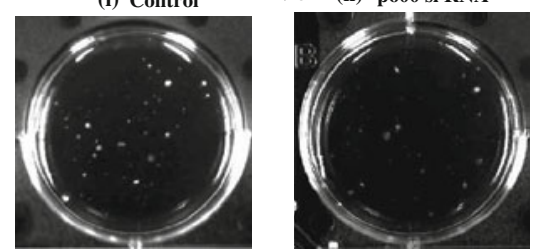

(iii) Colony numbers

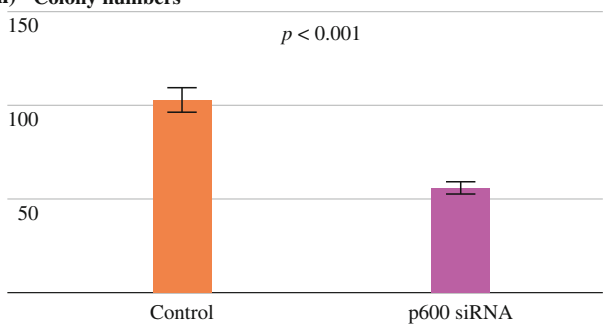

(e)

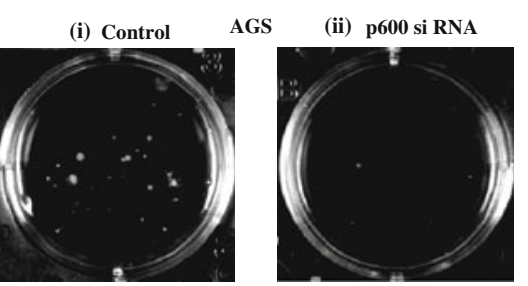

(iii) Colony numbers

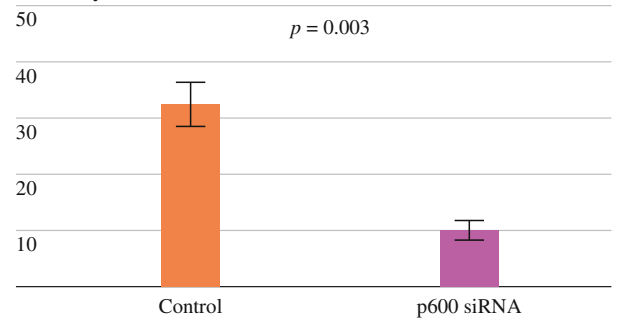

FIG. 4 Inhibition of p600 sensitizes gastric cancer cells to anoikis and reduces their viability under anchorage-independent conditions. a Cell viability assay under anchorage-independent conditions. The viability of (i) MKN45 and (ii) AGS gastric cancer cells were reduced by inhibition of p600 (MKN45, $P=0.033$; AGS, $P<0.001$ ). b Proportion of apoptotic (anoikis) cells under anchorage-independent conditions in (i) control siRNA-transfected MKN45 cells and (ii) p600-targeting siRNA-transfected MKN45 cells as analyzed by flow cytometry. c Proportion of apoptotic (anoikis) cells in (i) control

\section{Inhibition of p600 Suppresses the Establishment of Intraperitoneal Disseminated Tumors In Vivo}

Finally, we examined whether the inhibition of p600 suppressed the establishment of intraperitoneal disseminated tumors after intraperitoneal injection of MKN45 gastric cancer cells in vivo. Although both p600-targeting siRNA-transfected cells and control siRNA-transfected

siRNA-transfected AGS cells and (ii) p600-targeting siRNA-transfected AGS cells as analyzed by flow cytometry. d Macroscopic appearance of colonies formed in soft agar; $(i)$ control siRNAtransfected MKN45 cells and (ii) p600-targeting siRNA-transfected MKN45 cells. (iii) Number of colonies. e Macroscopic appearance of colonies formed in soft agar; (i) control siRNA-transfected AGS cells and (ii) p600-targeting siRNA-transfected AGS cells. (iii) Data graph. Data are expressed as mean \pm SD. The number of colonies was reduced by inhibition of p600 $(\mathrm{MKN} 45, P<0.001$; AGS, $P=0.003)$

cells developed disseminated nodules within the abdominal cavity, the number of nodules was significantly decreased by inhibition of p600 (Fig. 5; $P<0.001$ ). There was no statistically significant difference in the size of the disseminated nodules between mice injected with p600targeing siRNA-transfected cells and those injected with control siRNA-transfected cells (data not shown). These results suggest that p600 is involved in the establishment of 
FIG. 5 Inhibition of p600 suppresses the establishment of intraperitoneal disseminated tumors. a Macroscopic appearance of the intraperitoneal disseminated nodules; (i) control siRNAtransfected MKN45 cells injected into SCID mice, and (ii) p600-targeting siRNAtransfected MKN45 cells ( $n=10 /$ group). Arrows indicate intraperitoneal disseminated nodules. (iii) Resected disseminated nodules from SCID mice. b Data graph. Data are expressed as mean $\pm \mathrm{SD}$. The number of intraperitoneal disseminated nodules was significantly reduced by p600 inhibition $(P<0.001)$

(a)

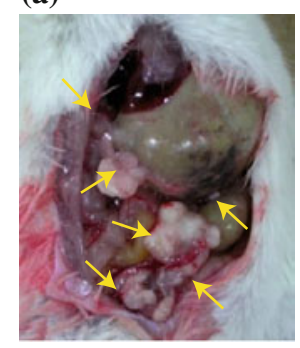

(i) Control

(iii)

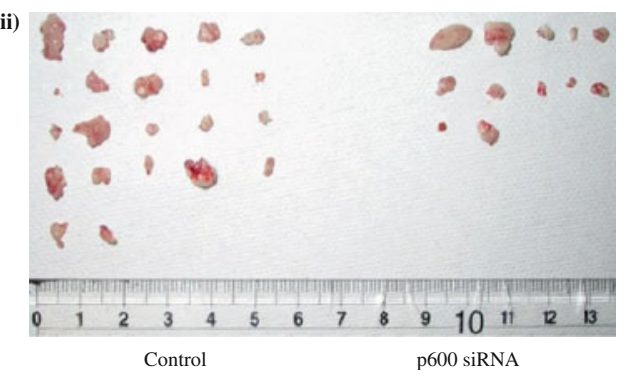

(b)

Disseminated nodules 30
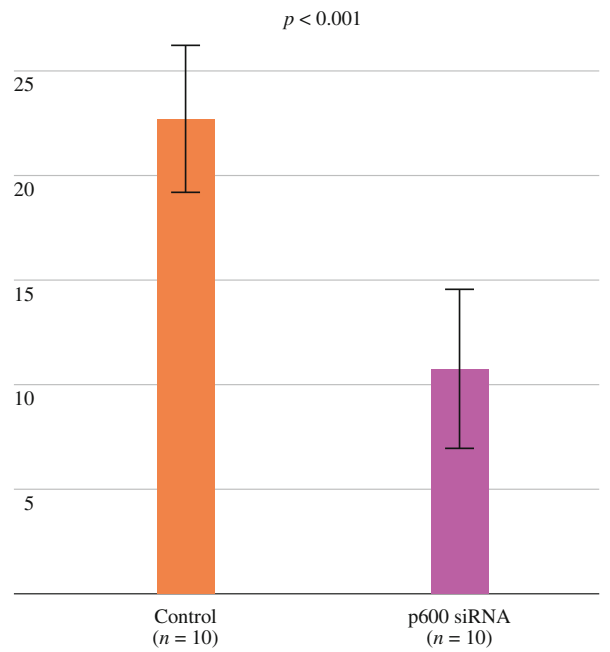

intraperitoneal disseminated gastric tumors, but not in anchorage-dependent cell growth in vivo.

\section{DISCUSSION}

In this study, we found that p600 expression was higher in gastric cancer cells than in normal gastric mucosal cells. Also, our in vitro experiments showed that both the invasiveness and anoikis resistance of gastric cancer cells were decreased by inhibition of p600. Furthermore, our in vivo experiments showed that the establishment of intraperitoneal disseminated gastric tumors was suppressed by inhibition of p600.

As far as we know, there are no reports investigating the differences of p600 expression levels between cancer cells and normal cells in clinical samples. Therefore, we investigated the expression of p600 mRNA in gastric cancer cells and compared it with that in normal gastric mucosal cells. As the result, p600 mRNA was statistically significantly upregulated in gastric cancer cells compared with normal gastric mucosal cells. Out of six gastric cancer cell lines, the five cell lines established from metastatic sites exhibited higher levels of p600 expression than the cell line established from a primary site. Recent results in a xenogeneic breast cancer model showed that the level of p600 expression was higher in metastatic tumors than in primary tumors. ${ }^{14}$ This supports our results, suggesting that p600 is involved in the progression of gastric cancer.

The first step in metastasis involves migration of cancer cells to, and invasion through, the basement membrane. ${ }^{8}$ In our study, inhibition of p600 decreased the number of invaded cells and the expression level of MMP-7 in both gastric cancer cell lines (MKN45 and AGS). Furthermore, inhibition of p600 induced overexpression of E-cadherin in MKN45 cells. A previous study reported that MMP-7 induced an epithelial-mesenchymal transition-like process in tumor cells by causing the shedding E-cadherin protein. ${ }^{26}$ Although further study of the relationship between p600, MMP-7, and E-cadherin is needed, it is possible that inhibition of p600 reduces invasiveness though the inhibition of epithelial-mesenchymal transition-like processes.

Once they have migrated through the basement membrane, gastric cancer cells in the abdominal cavity and/or the circulatory and lymphatic systems have to remain viable without contacting the ECM. They then have to implant at a distant site and initiate tumor growth. ${ }^{8}$ Our data showed that gastric cancer cells were sensitized to anoikis and that their colony-formation capacity was suppressed under anchorage-independent conditions by inhibition of p600. Previous studies have shown that activation of focal adhesion kinase plays a central role in anoikis, and that its activation is inhibited by the inhibition of p600. ${ }^{11,27,28}$ Therefore, p600 may be an important regulator of anoikis resistance in gastric cancer, although further studies are required to investigate this mechanism.

For the in vivo experiments, we injected gastric cancer cells into the peritoneal cavity of SCID mice and found that the establishment of intraperitoneal disseminated tumors was statistically significantly suppressed by inhibition of p600. Peritoneal dissemination of gastric cancer cells is a multistep process comprising invasion into the serosa from the stomach, detachment from the primary site and movement into the peritoneal cavity, attachment to the distant peritoneum, invasion into the subperitoneal space, and proliferation. Because the process of movement from a primary site into the peritoneum was not represented, 
peritoneal dissemination was not exactly represented by our in vivo experiment. However, because establishment of peritoneal dissemination after detachment from the primary site is one of key processes in peritoneal dissemination of gastric cancer cells, our data suggest the possibility that peritoneal dissemination is inhibited by inhibition of p600.

In conclusion, our results show that p600 expression is higher in gastric cancer cells than in normal gastric mucosal cells, and that knockdown of p600 inhibits the invasiveness and anoikis resistance of gastric cancer cells. This implies that p600 has potential as a new molecular target of gastric cancer therapy. Gene therapy targeting p600 might have a major effect on the processes involved in tumor progression, such as tumor invasion, metastasis, and dissemination, rather than in initiation and proliferation of tumors. Therefore, it is expected that the effects of p600 inhibition on gastric cancer would be best seen when used in combination with other treatments such as chemotherapy, although further study of the mechanism underlying the action and function of p600 is required.

ACKNOWLEDGMENT Supported in part by a Grant-in-Aid from the Ministry of Education, Culture, Sports, Science and Technology of Japan, and grants from the Japanese Society of Gastroenterology and Pancreas Research Foundation of Japan.

DISCLOSURE The authors declare no conflict of interest.

Open Access This article is distributed under the terms of the Creative Commons Attribution Noncommercial License which permits any noncommercial use, distribution, and reproduction in any medium, provided the original author(s) and source are credited.

\section{REFERENCES}

1. Parkin DM, Bray F, Ferlay J, Pisani P. Global cancer statistics, 2002. CA Cancer J Clin. 2005;55:74-108.

2. Alberts SR, Cervantes A, van de Velde CJ. Gastric cancer: epidemiology, pathology and treatment. Ann Oncol. 2003;14(Suppl 2):ii31-6

3. Glimelius B, Ekstrom K, Hoffman K, et al. Randomized comparison between chemotherapy plus best supportive care with best supportive care in advanced gastric cancer. Ann Oncol. 1997;8:163-8.

4. Hill ME, Cunningham D. Medical management of advanced gastric cancer. Cancer Treat Rev. 1998;24:113-8.

5. Hohenberger P, Gretschel S. Gastric cancer. Lancet. 2003; 362(9380):305-15.

6. Wohrer SS, Raderer M, Hejna M. Palliative chemotherapy for advanced gastric cancer. Ann Oncol. 2004;15:1585-95.

7. Fidler IJ. The pathogenesis of cancer metastasis: the "seed and soil" hypothesis revisited. Nat Rev Cancer. 2003;3:453-8.

8. Simpson CD, Anyiwe K, Schimmer AD. Anoikis resistance and tumor metastasis. Cancer Lett. 2008;272:177-85.

9. Frisch SM, Francis H. Disruption of epithelial cell-matrix interactions induces apoptosis. J Cell Biol. 1994;124:619-26.

10. Huh KW, DeMasi J, Ogawa H, et al. Association of the human papillomavirus type 16 E7 oncoprotein with the 600-kDa retinoblastoma protein-associated factor, p600. Proc Natl Acad Sci USA. 2005;102:11492-7.

11. Nakatani Y, Konishi H, Vassilev A, et al. p600, a unique protein required for membrane morphogenesis and cell survival. Proc Natl Acad Sci USA. 2005;102:15093-8.

12. DeMasi J, Huh KW, Nakatani Y, et al. Bovine papillomavirus E7 transformation function correlates with cellular p600 protein binding. Proc Natl Acad Sci USA. 2005;102:11486-91.

13. DeMasi J, Chao MC, Kumar AS, Howley PM. Bovine papillomavirus E7 oncoprotein inhibits anoikis. J Virol. 2007;81: 9419-25.

14. Montel V, Huang TY, Mose E, et al. Expression profiling of primary tumors and matched lymphatic and lung metastases in a xenogeneic breast cancer model. Am J Pathol. 2005;166: 1565-79.

15. Vasseur S, Malicet C, Calvo EL, et al. Gene expression profiling of tumours derived from rasV12/E1A-transformed mouse embryonic fibroblasts to identify genes required for tumour development. Mol Cancer. 2005;4:4.

16. Ohuchida K, Mizumoto K, Ishikawa N, et al. The role of S100A6 in pancreatic cancer development and its clinical implication as a diagnostic marker and therapeutic target. Clin Cancer Res. 2005;11:7785-93.

17. Tachikawa T, Irie T. A new molecular biology approach in morphology: basic method and application of laser microdissection. Med Electron Microsc. 2004;37:82-8.

18. Ohuchida K, Mizumoto K, Ohhashi S, et al. Twist, a novel oncogene, is upregulated in pancreatic cancer: clinical implication of Twist expression in pancreatic juice. Int $J$ Cancer. 2007;120:1634-40.

19. Sato N, Maehara N, Mizumoto K, et al. Telomerase activity of cultured human pancreatic carcinoma cell lines correlates with their potential for migration and invasion. Cancer. 2001;91: 496-504.

20. Zhang L, Mizumoto K, Sato N, et al. Quantitative determination of apoptotic death in cultured human pancreatic cancer cells by propidium iodide and digitonin. Cancer Lett. 1999;142:129-37.

21. Wu CY, Wu MS, Chen YJ, et al. Clinicopathological significance of MMP-2 and TIMP-2 genotypes in gastric cancer. Eur J Cancer. 2007;43:799-808.

22. Zheng HC, Sun JM, Li XH, et al. Role of PTEN and MMP-7 expression in growth, invasion, metastasis and angiogenesis of gastric carcinoma. Pathol Int. 2003;53:659-66.

23. Zhang S, Li L, Lin JY, Lin H. Imbalance between expression of matrix metalloproteinase- 9 and tissue inhibitor of metalloproteinase- 1 in invasiveness and metastasis of human gastric carcinoma. World J Gastroenterol. 2003;9:899-904.

24. Park IK, Kim BJ, Goh YJ, et al. Co-expression of urokinase-type plasminogen activator and its receptor in human gastric-cancer cell lines correlates with their invasiveness and tumorigenicity. Int J Cancer. 1997;71:867-73.

25. Lee KH, Choi EY, Hyun MS, et al. Association of extracellular cleavage of E-cadherin mediated by MMP-7 with HGF-induced in vitro invasion in human stomach cancer cells. Eur Surg Res. 2007;39:208-15.

26. Shibata S, Marushima H, Asakura T, et al. Three-dimensional culture using a radial low bioreactor induces matrix metalloprotease 7-mediated EMT-like process in tumor cells via TGFbeta1/ Smad pathway. Int J Oncol. 2009;34:1433-48.

27. Frisch SM, Vuori K, Ruoslahti E, Chan-Hui PY. Control of adhesion-dependent cell survival by focal adhesion kinase. J Cell Biol. 1996;134:793-9.

28. Liu G, Meng X, Jin Y, et al. Inhibitory role of focal adhesion kinase on anoikis in the lung cancer cell A549. Cell Biol Int. 2008;32:663-70 